\title{
Abdominal Aortic Aneurysms and Q Fever: An Odd Connection to Keep in Mind
}

\author{
Pedro Novais Lima ${ }^{\mathrm{a}, \mathrm{d}}$, Ruben Carvalho ${ }^{\mathrm{b}}$, Vania Constancio ${ }^{\mathrm{a}}$, Joana Cruz Silva ${ }^{\mathrm{a}}$, Celso Nunes ${ }^{\mathrm{a}}$, \\ Eduardo Silva ${ }^{\mathrm{a}}$, Anabela Goncalves ${ }^{\mathrm{a}}$, Manuel Fonseca ${ }^{\mathrm{a}}$, Luis F. Antunes ${ }^{\mathrm{a}, \mathrm{c}}$
}

\begin{abstract}
Abdominal aortic aneurysms (AAAs) secondary to Coxiella burnetii infections are rare but carry important implications. The diagnosis is difficult due to unspecific symptoms. To the authors' knowledge, this is the first AAA caused by chronic Q fever treated with an endoprosthesis.
\end{abstract}

Keywords: Q fever; Coxiella burnetii; Abdominal aortic aneurysm; Aortitis; Endovascular aneurysm repair

\section{Introduction}

Coxiella burnetii is an intracellular bacterium responsible for $\mathrm{Q}$ fever, a zoonotic infection more prevalent in rural areas. Acute infection usually manifests as a self-limited febrile illness, but more severe presentation like pneumonia or hepatitis may occur. Chronic vascular infection is less frequent than chronic endocarditis, and only a few cases of infra-renal abdominal aortic aneurysms (AAAs) in the context of Q fever have been reported [1]. Since the symptoms of acute infection may be unspecific, this entity is probably underdiagnosed.

The authors present a case of an AAA due to a chronic Coxiella burnetii infection managed using endovascular repair.

\section{Case Report}

\section{Investigations}

A 65-year-old male patient, with a past history of sarcoido-

Manuscript submitted July 30, 2021, accepted August 13, 2021

Published online August 25, 2021

${ }^{a}$ Angiology and Vascular Surgery Department, Centro Hospitalar e Universitario de Coimbra, Praceta Professor Mota Pinto, 3004-561 Coimbra, Portugal ${ }^{b}$ Infectious Diseases Department, Centro Hospitalar e Universitario de Coimbra, Praceta Professor Mota Pinto, 3004-561 Coimbra, Portugal

'Faculty of Medicine, University of Coimbra, Rua Larga 2, 3000-370 Coimbra, Portugal

${ }^{\mathrm{d} C o r r e s p o n d i n g ~ A u t h o r: ~ P e d r o ~ N o v a i s ~ L i m a, ~ A n g i o l o g y ~ a n d ~ V a s c u l a r ~ S u r g e r y ~}$ Department, Centro Hospitalar e Universitario de Coimbra, Praceta Professor Mota Pinto, 3004-561 Coimbra, Portugal. Email: pedro_lima20@hotmail.com

doi: https://doi.org/10.14740/jmc3760 sis, presented with an AAA detected on a routine abdominal ultrasound. Several abdominal ultrasounds performed in the previous years had never detected any AAA. Along with sarcoidosis (histologically diagnosed on a cervical adenopathy biopsy), he had arterial hypertension and atrial fibrillation. The patient was compliant to telmisartan $40 \mathrm{mg}$ q.d. and apixaban $5 \mathrm{mg}$ b.i.d. No symptoms were apparent. The patient had an excellent functional status and lived an active lifestyle. No fever, chills, malaise, fatigue or loss of weight were to be reported. He reported no domestic or professional contact with animals.

\section{Diagnosis}

A computed tomography angiography (CTA) confirmed the ultrasound finding, revealing a saccular infra-renal AAA with $51 \mathrm{~mm}$ of diameter (Fig. 1a).

Blood cell counts and biochemistry surveys, including the erythrocyte sedimentation rate and C-reactive protein, were within normal values. Serologic studies were negative, except for Coxiella burnetii IgG antibodies at a 1:1,280 titer. A minimal burden of atherosclerosis throughout the thoracoabdominal aorta, along with the saccular morphology, prompted an investigation for aortitis etiologies. An ${ }^{18}$ fluorodeoxyglucosepositron emission tomography/computed tomography $\left({ }^{18} \mathrm{FDG}-\right.$ $\mathrm{PET} / \mathrm{CT}$ ) was performed but only para-aortic adenopathies had a significant uptake (Fig. 1b).

Further indirect immunofluorescence (IIF) testing (performed at Instituto Nacional de Saude Doutor Ricardo Jorge) revealed high titers of both anti-phase II and anti-phase I IgG antibodies (1:3,200 and 1:1,600, respectively). Anti-phase II and anti-phase I IgM antibodies were slightly above the positivity cutoff. Polymerase chain reaction (PCR) detection of Coxiella burnetii was not performed. No cardiac valvular vegetations were detected in the transthoracic echocardiography.

\section{Treatment}

The patient promptly started doxycycline $100 \mathrm{mg}$ b.i.d. and hydroxychloroquine $200 \mathrm{mg}$ t.i.d., and after 10 days he was submitted to an endovascular aortic repair (EVAR) with a bifurcated Excluder ${ }^{\circledR}$ endoprosthesis (W. L. Gore \& Associates, Inc., Flagstaff, AZ, USA).

He had an uneventful recovery and was discharged at the 

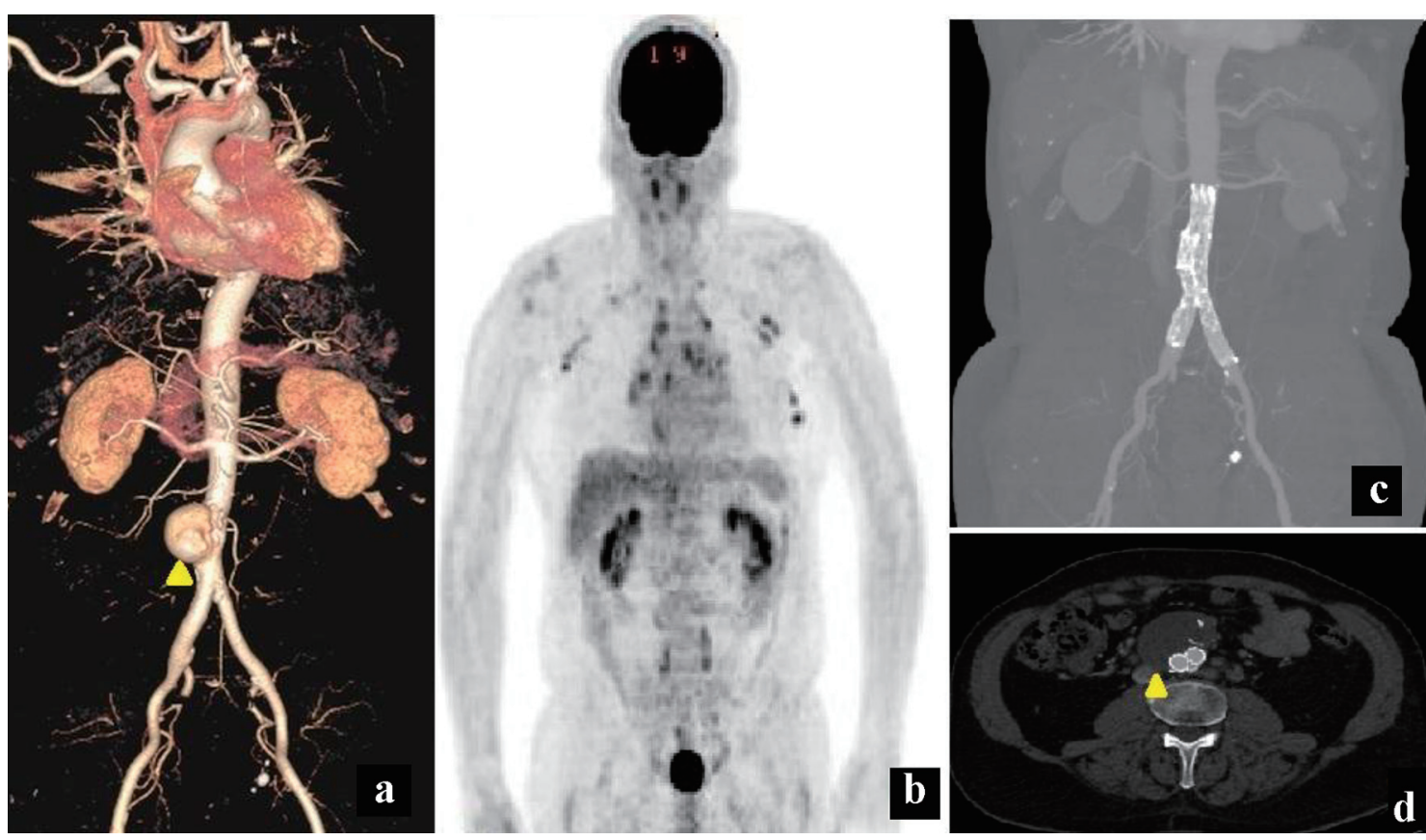

b

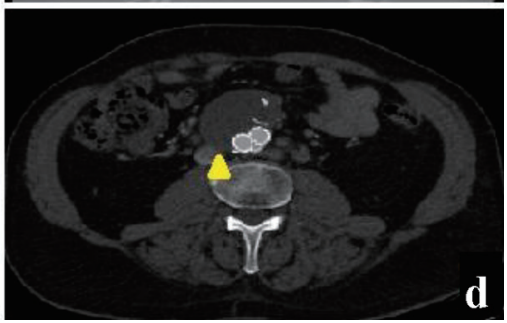

Figure 1. (a) A computed tomography angiogram (CTA) 3D reconstruction revealing a saccular infra-renal abdominal aortic aneurysm (AAA) (yellow arrowhead). (b) An ${ }^{18}$ fluorodeoxyglucose-positron emission tomography (18FDG-PET) image depicting paraaortic and mediastinal adenopathies. No abdominal aortic uptake was noticed. (c) A 1 month post-operative CTA on a maximal intensity projection reconstruction showing permeability of the endograft used to treat the aneurysm. No contrast opacification was visible within the aneurysm sac. (d) An axial projection of the same CTA as (c). During arterial phase acquisition, no contrast opacification was visible inside the aneurysm sac (yellow arrowhead).

third post-operative day.

\section{Follow-up and outcomes}

The antibiotic therapy was maintained for 1 year with no side effects, and is intended to be administered for at least 18 months. During follow-up, a complete aneurysm thrombosis was noticed (Fig. 1c, d) and no signs of graft infection were present.

\section{Discussion}

Q fever is a zoonosis caused by Coxiella burnetii. It is endemic with a large reservoir in wild and domestic animals. Acute infection usually manifests as a self-limited febrile illness, so its incidence is probably underestimated [2]. Chronic Q fever has a variety of manifestations including endocarditis and infection of vascular prosthesis. The diagnosis of this condition is challenging, and in most cases, the confirmation is serologic.

Cardiovascular chronic infection occurs most frequently as culture-negative endocarditis, and vascular manifestations like aortic aneurysms are rare. According to de Worm et al [3], around 230 cases of $Q$ fever vascular infection have been reported with both native aortitis and vascular graft infections described.

Atherosclerosis is considered as a potential risk for development of Coxiella aortitis, since plaques and thrombus represent a nidus for bacterial ingrowth and account as sites of immunodeficiency [4].
In this case, the aneurysm was likely a consequence of the chronic infection and not the other way around. Despite the patient's cardiovascular risk factors, the aorta was minimally atherosclerotic. The saccular morphology of the AAA was also consistent with a non-degenerative cause. Furthermore, the patient had several abdominal ultrasound examinations performed in the years preceding the diagnosis and the AAA was never detected.

Phase I and phase II IgG titers were above 1:800 and 1:1,600, respectively, suggesting a chronic Coxiella burnetii infection. The antibiotic regimen used is effective for $\mathrm{Q}$ fever endocarditis and other forms of chronic $\mathrm{Q}$ fever $[5,6]$.

Adjunctive ${ }^{18} \mathrm{FDG}-\mathrm{PET} / \mathrm{CT}$ is usually helpful for diagnostic purposes but the results should be interpreted carefully. In several reports, this imaging technique is described for both native aortic and vascular graft infections, with no distinction between these entities. Positive findings can be misled by confounding factors such as bacterial co-infection [7] or severe Q fever presentations. In a recent paper, reporting patients with a positive PCR test for Coxiella burnetii, ${ }^{18} \mathrm{FDG}$-PET/CT allowed detection of vascular infection in only $50 \%$ of cases [8]. None of those patients had evidence of spondylodiscitis or abcesses.

The optimal therapy includes resection of the aneurysm, debridement of infected surrounding tissue and reconstruction using autologous materials. In this case, an endovascular approach was preferred for several reasons. The patient was asymptomatic as opposed to the majority of cases described in the literature. In a review conducted by Sessa et al (2005), from the 13 cases included, 53\% had fever. Several reports also describe concomitant abcesses [9], vertebral osteomyelitis 
[10], aortocaval [11] or aorto-digestive fistulae [12].

In this patient, the ${ }^{18} \mathrm{FDG}-\mathrm{PET} / \mathrm{CT}$ revealed no significant inflammatory activity around the aneurysm. Since the aneurysm was located near the aortic bifurcation and considering that open repair would imply a total aneurysmectomy, the patient would be at a higher risk of intra-operative lesion of the ureters, caval bifurcation and lumbar splanchnic nerves (potentially leading to sexual dysfunction).

The choice of aortic graft was also made considering the infra-renal fixation offered by Excluder ${ }^{\circledR}$ devices, in case of future need for prosthetic explantation.

During follow-up, no complications or signs of graft infection have been noticed after 1 year. The treatment plan includes continuous antibiotic therapy for at least 18 months.

\section{Learning points}

Vascular chronic Q fever is a difficult entity to diagnose but may have important implications upon a patient's life. Physician awareness about its existence, risk factors and adjunctive imaging tools may increase the number of detected cases.

The clinical presentation, affected aortic segment and patient's fitness for surgery should all be taken into consideration to offer the best patient-centered therapy. Endovascular therapy can be safely performed and may be considered if lowgrade infection is present or as a "bridging" strategy.

\section{Acknowledgments}

None to declare.

\section{Financial Disclosure}

No funding has been received from any entity to the development of the present paper.

\section{Conflict of Interest}

No conflict of interest is to be reported from any of the authors.

\section{Informed Consent}

The patient has given a written consent to include medical information.

\section{Author Contributions}

Case study: PNL, RC, and LA. Case treatment and data collection: PNL, LA, and MF. Paper writing: PNL and RC. Review: $\mathrm{VC}, \mathrm{JCS}, \mathrm{CN}, \mathrm{ES}$, and AG.

\section{Data Availability}

Any inquiries regarding supporting data availability of this study should be directed to the corresponding author.

\section{References}

1. Sessa C, Vokrri L, Porcu P, Maurin M, Stahl JP, Magne JL. Abdominal aortic aneurysm and Coxiella burnetii infection: report of three cases and review of the literature. J Vasc Surg. 2005;42(1):153-158.

2. Fournier PE, Casalta JP, Piquet $P$, Tournigand $P$, Branchereau A, Raoult D. Coxiella burnetii infection of aneurysms or vascular grafts: report of seven cases and review. Clin Infect Dis. 1998;26(1):116-121.

3. de Worm S, Giot JB, Courtoy C, Gillet E, Amrane S, Huynen P, Van Esbroeck M, et al. A case of giant cell arteritis associated with culture-proven Coxiella burnetii aortitis. Int J Infect Dis. 2018;69:50-54.

4. Riga C, Bicknell C, Jindal R, Cheshire N, Hamady M. Endovascular stenting of peripheral infected aneurysms: a temporary measure or a definitive solution in high-risk patients. Cardiovasc Intervent Radiol. 2008;31(6):12281235.

5. Raoult D. Chronic Q fever: expert opinion versus literature analysis and consensus. J Infect. 2012;65(2):102108.

6. Million M, Raoult D. Recent advances in the study of Q fever epidemiology, diagnosis and management. J Infect. 2015;71(Suppl 1):S2-9.

7. Merhej V, Cammilleri S, Piquet P, Casalta JP, Raoult D. Relevance of the positron emission tomography in the diagnosis of vascular graft infection with Coxiella burnetii. Comp Immunol Microbiol Infect Dis. 2012;35(1):45-49.

8. Hagenaars JC, Wever PC, Vlake AW, Renders NH, van Petersen AS, Hilbink M, de Jager-Leclercq MG, et al. Value of 18F-FDG PET/CT in diagnosing chronic Q fever in patients with central vascular disease. Neth J Med. 2016;74(7):301-308

9. Jayet J, Raux M, Allaire E, Desgranges P, Cochennec F. Treatment of an abdominal aortic aneurysm infected by coxiella burnetii using a cryopreserved allograft. Ann Vasc Surg. 2016;33:227 e229-227 e212.

10. Mongardon N, Dubory A, Dorget A, De Wailly G, Lepeule R, Cochennec F. Vascular infection and vertebral destruction in a patient with Q fever. Lancet Infect Dis. 2018;18(2):226.

11. Prinsen JH, Boersma D, van Loenhout R, van Schaik PM, Verhoeven BA. Persistent endoleak after endovascular aneurysm repair for acute Q-fever-infected aortocaval fistula. Vascular. 2015;23(6):645-647.

12. Eldin C, Mailhe M, Lions C, Carrieri P, Safi H, Brouqui $\mathrm{P}$, Raoult D. Treatment and prophylactic strategy for coxiella burnetii infection of aneurysms and vascular grafts: a retrospective cohort study. Medicine (Baltimore). 2016;95(12):e2810. 